\title{
Target differentiation and localization using infrared sensors
}

\author{
Tayfun Aytaç* and Billur Barshan \\ Department of Electrical and Electronics Engineering \\ Bilkent University, Bilkent, TR-06533 Ankara, Turkey \\ E-mail:\{taytac, billur\}@ee.bilkent.edu.tr
}

\begin{abstract}
We discuss the use of low-cost infrared sensors in differentiating and localizing commonly encountered target primitives in indoor environments, such as planes, corners, edges, and cylinders. Single intensity readings are highly dependent on target location and properties and this dependence cannot be represented simply. We propose a method that can achieve position-invariant target differentiation without relying on absolute intensity readings and verify it experimentally. The correct identification rates for planes, $90^{\circ}$ corners and edges, and cylinders are $90 \%, 100 \%, 82.5 \%$, and $92.5 \%$, respectively. The distance of the target can be estimated with an average error of $0.59 \mathrm{~cm}$ and the azimuth angle can be estimated with an error of $1.58^{\circ}$.
\end{abstract}

\section{INTRODUCTION}

Infrared sensors are inexpensive, practical and widely available devices. However, it is difficult to make reliable distance estimates based on the value of a single intensity return because the return depends on both the geometry and the surface properties of the reflecting target. Likewise, the geometry or surface properties cannot be deduced from simple intensity returns without knowing the distance and angular location of the target. In this paper, we propose a scanning technique and an algorithm that can determine the type of the target in a manner which is invariant to its location. Once the target type is determined, its distance and azimuth angle can be estimated. Target identification is of great interest especially in robotics applications, where there is need to identify targets in mobile robot environments for autonomous operation. Typical applications of infrared sensors in this area are mainly floor sensing, navigational referencing, and collision avoidance at short ranges. ${ }^{1}$ Infrared sensors have been used to locate edges of doorways in mobile robot navigation. ${ }^{2}$ In another study, the surface properties of an object at a known distance have been determined using the Phong model, and using this information, the infrared sensor employed has been modeled as an accurate range finder for surfaces at short ranges ${ }^{3}(3-25 \mathrm{~cm})$. However, to the best of our knowledge, no attempt has been made to differentiate targets using infrarcd sensors.

\section{THE METHOD}

The infrared sensor 4 used in this study works with 20-28 V DC input voltage, and provides analog output voltage proportional to the measured intensity between ranges $0-60 \mathrm{~cm}$. The detector window is covered with an infrared filter to minimize the effect of ambient light on the intensity measurements. First, we have attempted to achieve target diffcrentiation with a single infrarcd scnsor. Our method is based on angularly scanning the targct over a certain angular range. We have shown that the resulting angular intensity scans contain sufficient information to identify several different target types and estimate their distance and azimuth. Reference data sets are collected for each target with $2.5 \mathrm{~cm}$ distance increments, making $0^{\circ}$ with the line-of-sight of the experimental setup. We tested our method by locating the targets at randomly selected distances and azimuth angles and collecting 120 such test scans. With this approach, $93 \%$ average correct classification rate, and distance and azimuth estimation errors of $0.59 \mathrm{~cm}$ and $1.69^{\circ}$ are achieved. However, with the use of a single sensor, it would be necessary to store reference data for different types of surfaces. To remedy this, we considered the use of two sensors mounted on a 12 inch rotary table horizontally, with a center-to-center separation of $11 \mathrm{~cm}$. Typical scan patterns for this configuration arc shown in Fig 1. Based on thesc pattcrns, it is obscrved that the return signal intensity patterns for a corner, which have two maxima and a single minimum, differ significantly from those of other targets which have a single maximum [Fig. 1.a]. Because of these distinctive characteristics, the corner differentiation rule is first employed. If the target is found not to be a corner, we next check whether it

${ }^{*}$ Corresponding Author. 

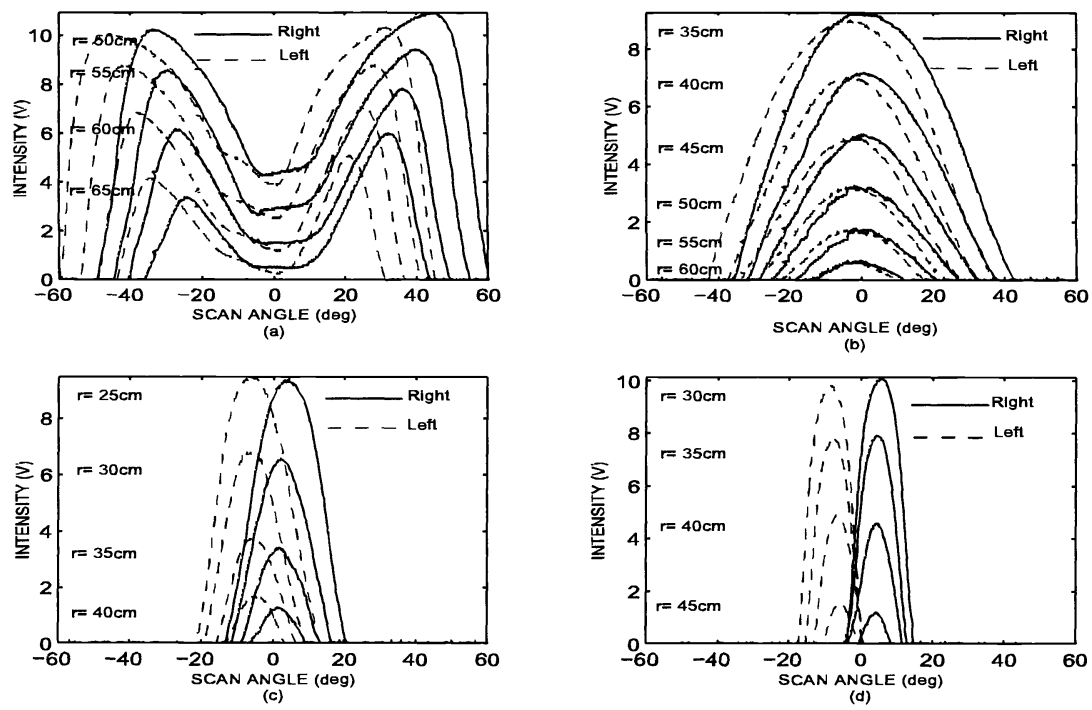

Figure 1. Intensity vs. scan angle characteristics for various targets making $0^{\circ}$ with the line-of-sight of the experimental setup: (a) corner, (b) plane, (c) edge, and (d) cylinder.

is a plane by comparing the absolute difference of angle values at which the two intensity patterns have their maxima as shown in Fig. 1.a. If the target is also not a plane, to check whether it is an edge or a cylinder, we use the ratio of the maximum intensity to the intensity value at the intersection point, if the patterns intersect [Fig. 1.c and d]. If the two intensity patterns do not intersect, the target is classified as undetermined, although this situation did not arise in our tests.

\section{RESULTS}

With the experimental setup described in Section 2, the algorithm is tested with a plane, $90^{\circ}$ corner and edge, and a cylinder of radius $48 \mathrm{~mm}$ using 160 test cases. A target confusion matrix, which displays the information about the actual and detected targets by the sensors, is presented in Table 1. The algorithm differentiates corners with $100 \%$ accuracy. Furthermorc, when the target is not a corner, it is never classificd as a corner.

Table 1: Target confusion matrix. P:plane, C:corner, E:edge, CY:cylinder, U:undetermined.

\begin{tabular}{|c|c|c|c|c|c|c|}
\hline TARGET & \multicolumn{5}{|c|}{ DIFFERENTIATION RESULT } & TOTAL \\
\hline & $\bar{P}$ & $\overline{\mathrm{C}}$ & $\overline{\mathrm{E}}$ & $\overline{C Y}$ & $\overline{\mathrm{U}}$ & \\
\hline $\mathrm{P}$ & 36 & - & 4 & - & - & 40 \\
\hline $\mathrm{C}$ & 0 & 40 & - & - & - & 40 \\
\hline $\mathrm{E}$ & 4 & - & 33 & 3 & - & 40 \\
\hline $\mathrm{CY}$ & 3 & - & - & 37 & - & 40 \\
\hline $\mathrm{U}$ & - & - & - & - & - & - \\
\hline TOTAL & 43 & $\overline{440}$ & 37 & 40 & - & 160 \\
\hline
\end{tabular}

\section{CONCLUSION}

The accomplishment of this study is that even though the intensity patterns are highly dependent on target range and azimuth, and this dependence cannot be represented by a simple relationship, by developing a method that does not rely on absolute intensity values, we effectively achieve position-invariant target differentiation. An average correct target differentiation rate of $91 \%$ is achieved.

\section{REFERENCES}

1. H.R. Everett, Sensors for Mobile Robots, Theory and Application (A K Peters, Wellesley, 1995).

2. A.M. Flynn, Int. J. Robot. Res., 17, 6, pp.598 623 (1988).

3. P.M. Novotny and N.J. Ferrier, Proc. IEEE Int. Conf. Robot. Automat., 2, pp.1644 1649 (1999).

4. IRS-U-4A/20-28 V, Datasheet Matrix Elektronik AG, Kirchweg 24 CH-5422 Oberehrendingen, Switzerland. 